\title{
A decade of PALSEA: Advances and future aims
}

\author{
Natasha L.M. Barlow', G.A. Milne ${ }^{2}$ and J.D. Shakun ${ }^{3}$
}

In 2019, the PAGES and INQUA-CMP (inqua. org/commissions/cmp) working group PALeo constraints on SEA level rise (PALSEA) entered its third phase. Established in 2008 by Mark Siddall and colleagues (Siddall et al. 2009), the second phase of PALSEA (PALSEA2, 2013-2017) was led by Anders Carlson, Andrea Dutton, Antony Long and Glenn Milne. The group continued the successful approach of bringing together observational and modeling scientists focusing on ice-sheet, climate and sea-level change to better define constraints on ice-sheetdriven paleo sea-level rise and apply this knowledge to inform projections of future change. Increased dialogue between these, and broader, communities over the past 10 years of PALSEA have resulted in over 100 peer-reviewed publications tackling societally important questions as to the drivers of ice-sheet and sea-level change. The consequences have been far-reaching, with a key indicator being the significant paleo sea-level and ice-sheet components included in the IPCC AR5 report (Church et al. 2013; Masson-Delmotte et al. 2013), and several members of the PALSEA community currently working as authors of the IPCC AR6 report.

One standout contribution of the PALSEA working group has been the considerable progress made by the observational community to agree on best practice for methods to reconstruct the elevation of former sea levels (Khan et al. p. 10). This has resulted in the production of a standardized sea-level database protocol (Düsterhus et al. 2016;
Khan et al. p. 10) and a growing number of high-quality regional and global databases for different time periods (Miller et al. p. 4; Dutton and Barlow, p. 6; Barnett et al. p. 8; Khan et al. p. 10) that provide the foundation for empirical and process-based modeling studies which aim to identify the underlying driving mechanisms across a range of temporal and spatial scales.

Research stimulated by PALSEA has identified processes that complicate estimates of global mean sea level (GMSL), and thus ice volume, from a geographically distributed set of local releative sea level (RSL) reconstructions. This has led to ongoing efforts to improve our understanding and ability to more accurately model processes such as glacial isostatic adjustment (Milne et al. p. 16), mantle dynamic topography (Austermann and Forte, p. 18), sediment flux and associated loading (Ferrier et al. p. 24), and overprinting records of extreme storm or wave events (Engelhart et al. p. 26). As these models improve, so too will estimates of global ice volume during past warm periods, providing a target and stimulus for the data and modeling communities to determine the minimum extent of the Greenland and Antarctic ice sheets and thus the climate drivers of sea-level rise during key intervals of Earth's history (Carlson and Larsen. p. 12; Sime et al. p. 14; de Boer et al. p. 20; OttoBliesner et al. p. 22).

Late Holocene RSL research (Barnett et al. p. 8 ) has shown that an ability to constrain rates of sea-level change can provide important

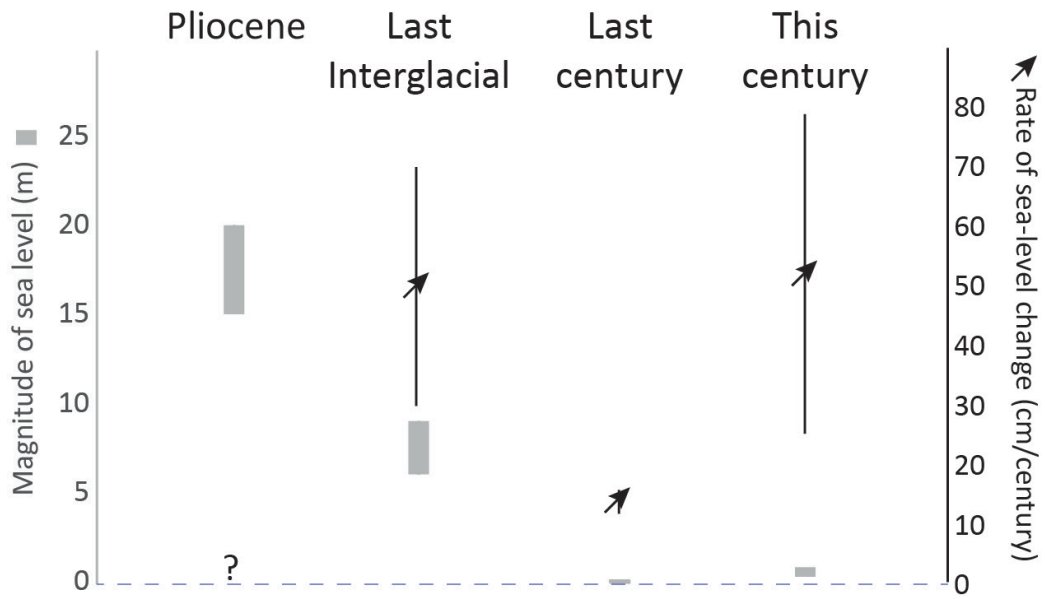

Figure 1: Summary of the magnitude of GMSL highstand during the Pliocene (Miller et al. p. 4), the Last Interglacial (Dutton and Barlow, p. 6; Dutton et al. 2015) relative to present, the last century relative to preindustrial (1900 CE; Barnett et al. p.8; Kopp et al. 2016) values, and this century relative to the year 2000 (Church et al. 2013). The rate of GMSL change during the Pliocene is not well constrained; LIG estimate based upon the "likely" range of 3-7 m/kyr from Kopp et al. (2013); rate for the last century based upon the year 1900-2000 rate from Kopp et al. (2016); and the rate for this century based upon the average rates of GMSL rise for the 21st century using the projected rise for the period 1986-2005 to 2081-2100 as given in Table 13.5 of Church et al. (2013) using the "likely" ranges for RCPs 2.6 and 8.5 (specifically, the lower bound for RCP 2.6 and upper bound for RCP 8.5).

\section{P A L S E A (2) PALSEA}

insights into drivers, synchronicity, and feedbacks in the coupled Earth system. However, prior to the radiocarbon dating window (ca. $>40$ kyr BP), establishing rates of both ice-sheet and sea-level change continues to be a challenge (Miller et al. p. 4; Dutton and Barlow, p. 6). While community efforts to better quantify GMSL have been successful, constraining rates of change during past warm periods remains an elusive target (Fig. 1), but a critical one to more effectively use paleo observations in future projections (Horton et al. p. 28). The spatial aspect of the problem has also been brought to the fore though PALSEA activities. Sea-level change is not globally uniform and so there is now a clear shift towards developing and understanding regional sea-level changes and relating these to GMSL.

The new phase of PALSEA, led by researchers who have benefited from the PALSEA network as early-career scientists (Jacqueline Austermann, Natasha Barlow, Alessio Rovere and Jeremy Shakun), seeks to build on past success and focus efforts on moving towards some of the outstanding goals outlined above. As the contemporary system exhibits accelerated and potentially irreversible changes (e.g. Shepherd et al. 2018), the relevance of the paleo record, which contains information on such responses in the past, becomes even more critical (Horton et al. p. 28). This Past Global Changes Magazine brings together contributions that summarize the state-of-the-science following the last decade of investigation and collaboration, as well as provide a stimulus into critical areas of research for PALSEA and the wider community.

\section{AFFILIATIONS \\ 'School of Earth and Environment, University of Leeds, UK \\ 2Department of Earth and Environmental Sciences, University of Ottawa, Canada \\ ${ }^{3}$ Department of Earth and Environmental Sciences, Boston College, USA}

\section{CONTACT}

Natasha Barlow: N.L.M.Barlow@leeds.ac.uk

\section{REFERENCES}

Church JA et al. (2013) In: Stocker TF et al. (Eds) Climate Change 2013: The Physical Science Basis, Cambridge University Press, 1137-1216

Düsterhus A et al. (2016) Clim Past 12: 911-921

Dutton A et al. (2015) Science 349: aaa4019

Kopp RE et al. (2013) Geophys J Int 193: 711-716

Kopp RE et al. (2016) Proc Natl Acad Sci 113: E1434-E1441 Masson-Delmotte V et al. (2013) In: Stocker TF et al. (Eds)

Climate Change 2013: The Physical Science Basis,

Cambridge University Press, 383-464

Shepherd A et al. (2018) Nature 558: 219-222

Siddall M et al. (2009) PAGES News 17: 49-88 